Helbig, Marko; Grießbach, Gert; Witte, Herbert; Schack, Bärbel:

\title{
Zeitvariante Bispektralanalyse auf der Basis einer adaptiv rekursiven
} Fouriertransformation

\footnotetext{
Zuerst erschienen in: $\quad$ Biomedizinische Technik = Biomedical Engineering. - Berlin [u.a.] : de Gruyter. - 47 (2002), S1b, S. 585-587.

Jahrestagung der Deutschen Gesellschaft für Biomedizinische Technik (DGBM) im VDE ; 36 (Karlsruhe) : 2002.09.25-27

Erstveröffentlichung: 2002

Datum Digitalisierung: $2009-11-26$

ISSN (online): $\quad$ 1862-278X

ISSN(print) 0013-5585

DOI: $\quad$ 10.1515/bmte.2002.47.s1b.585

[Zuletzt gesehen: 2019-12-06]
}

„Im Rahmen der hochschulweiten Open-Access-Strategie für die Zweitveröffentlichung identifiziert durch die Universitätsbibliothek IImenau."

"Within the academic Open Access Strategy identified for deposition by IImenau University Library."

„Dieser Beitrag ist mit Zustimmung des Rechteinhabers aufgrund einer (DFGgeförderten) Allianz- bzw. Nationallizenz frei zugänglich."

„This publication is with permission of the rights owner freely accessible due to an Alliance licence and a national licence (funded by the DFG, German

Research Foundation) respectively."

\section{DFG}

Nationallizenzen 


\title{
ZEITVARIANTE, BISPEKTRALANALYSE AUF DER BASIS EINER ADAP'TIV REKURSIVEN FOURIER'TRANSFORMATION
}

\author{
M. Helbig', G. Gricßbach', H. Witte², B. Schack ${ }^{2}$ \\ 'Institut lïr Bionecd. Technik u. Informatik, Technische Universität Ilmenau, Deutschland \\ 'Institut für Med. Statistik, Informatik u. Dokumentation, FSU Jena, Deutschland
}

Marko.Helbig@tu-ilmenau.de

\begin{abstract}
Ibstract - An appropriate investigation of quadratic phase' couplings: (QPC) in non-stationary signals requires time'-1'arumt me'thods of bispectral anulysis.

A me'w approach for time-variant estimation of power spectrum and bispectrum based on an adaptively, recursively estimuted Fourier transform (ADFT) is presented in this paper.

A reducied calculation effort and the possibility of the calculation of the bispectrum for selected frequency triples are important advantages of this method. Because of the recursi'e calculation, the ADFT is convenient for analying ongoing signals. This will be demonstrated for simulated and real biomedical signals.
\end{abstract}

Keywords - DFT, adaptive recursive estimation, timevariant spectral and bispectral analysis

\section{Einleitung}

Mit Hilfe der Bispektralanalyse ist es möglich, quadratische Phasenkopplungen (QPC) zwischen verschiedenen Frequenzkomponenten zu analysieren. Die klassische, auf ein festes Zeitintervall bezogene Bispektralanalyse setzt analog zur herkömmlichen Spektralanalyse Signalanforderungen (vor allem Stationarität) voraus, denen reale Biosignale zumeist nicht genügen. Transient auftretende quadratische Phasenkopplungen können mit den konventionellen Verfahren nicht analysiert werden. Zur Analyse dieser sich zeitlich ändernden Phasenkopplungsphänomene ist eine zeitvariante Bispektralanalyse mit einer ausreichend hohen Auflösung im Spektralbereich erforderlich. Eine neue Methode auf der Basis einer adaptiv rekursiven Fouriertransformation (ADFT) wird hierzu vorgestellt.

\section{Methoden}

\section{Adaptiv rekursive Schätzungen}

Die Grundlagen zu den adaptiv rekursiven Schätzverfahren wurden in [1] entwickelt und sind in verschiedenen Publikationen dargelegt [2].

Das Grundelement der im folgenden dargestellten adaptiv rekursiven Fouriertransformation stellt der adaptiv rekursive Mittelwertschätzer $\mathbf{M}^{\mathbf{C}}$ der diskreten Zeitreihe $\{x(k)\}_{k=0,1,2 \ldots . ., N-1}$ dar. $\mathbf{L}_{k}$ symbolisiert den Lagoperator und $c_{k}$ die Adaptionsvariable.

$$
\begin{array}{lc}
M^{\mathrm{c}}: & \mathbf{M}_{0}=\mathrm{m}_{0} \\
& \mathbf{M}_{\mathrm{k}+1}=\mathbf{M}_{\mathrm{k}}+\mathrm{c}_{\mathrm{k}} \cdot\left(\mathrm{x}_{\mathrm{k}+1}-\mathbf{M}_{\mathrm{k}}\right) \\
\mathbf{L}_{\mathrm{k}}: & \mathbf{L}_{\mathrm{k}}\left(\left\{\mathrm{x}_{\mathrm{j}}\right\}_{\mathrm{j}=0,1, \ldots}\right)=\left\{\mathrm{x}_{\mathrm{j}+\mathrm{k}}\right\}_{\mathrm{j}=0,1, \ldots}
\end{array}
$$

\section{Adaptiv rekursive Fouriertransformation (ADFT)}

Die diskrete Fouriertransformation (DFT) eines Signalabschnitts $\{x(k)\}_{k=0,1,2 \ldots . . . N-1}$ ist wie folgt definiert:

$$
X(n)=\sum_{k=0}^{N-1} x(k) \cdot e^{-j 2 \pi n k / N}, \quad n=0,1, \ldots, N-1
$$

Zur Vereinfachung der Schreibweise sei $W_{N}=e^{-j 2 \pi / N}$. Damit wird aus (2):

$$
X(n)=\sum_{k=0}^{N-1} x(k) \cdot W_{N}^{n k}, \quad n=0,1, \ldots, N-1
$$

Die Einheitswurzeln $W_{N}^{n k}$ besitzen eine Reihe von Eigenschaften (Periodizität, Symmetrie), die insbesondere im Algorithmus der FFT ausgenutzt werden. Im adaptiven Ansatz, der in dieser Arbeit vorgestellt wird, spielt die Struktur der Potenzierung des Drehfaktors $\mathrm{W}_{\mathrm{N}}$ die grundlegende Rolle. Ausgangspunkt ist die DFT-Berechnung innerhalb eines über das Signal $\{x(t)\}_{t=0,1,2, \ldots .}$ gleitenden N-Punkte-Analysefensters:

$$
X(n, t)=\sum_{k=0}^{N-1} x(t-N+1+k) \cdot W_{N}^{n k}, \quad n=0,1, \ldots, N-1
$$

Diese Berechnung kann auch rekursiv aus $X(n, t-1)$ erfolgen, wobei das Fenster schrittweise um jeweils ein Sample verschoben wird :

$$
X(n, t)=(X(n, t-1)-X(t-N)) / W^{n}+x(t) \cdot W^{n \cdot(N-1)}
$$

Der adaptiv rekursive Ansatz besteht darin, die Mittelung nach (5) durch die adaptiv rekursive Mittelwertschätzung zu ersetzen. Es resultiert die adaptiv rekursive Fouriertransformation: 


$$
\begin{aligned}
X(n, 0)= & c_{0} \cdot x(0) \cdot W^{n \cdot(N-1)} \\
X(n, t)= & \left(1-c_{t}\right) \cdot\left(X(n, t-1) / W^{n}\right)+c_{t} \cdot x(t) \cdot W^{n \cdot(N-1)} \\
= & X(n, t-1) / W^{n} \\
& +c_{t} \cdot\left(x(t) \cdot W^{n \cdot(N-1)}-X(n, t-1) / W^{n}\right)
\end{aligned}
$$

Zur Kennzeichnung wird folgendes Operator-Symbol eingeführt:

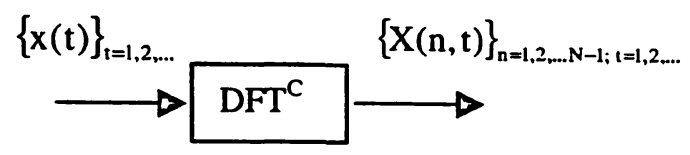

Zeitvariante Bispektralanalyse auf der Basis der ADFT Die klassischen Schätzverfahren des Bispektrums $B\left(n_{1}, n_{2}\right)$ für stationäre Signale basieren auf dem indirekten Ansatz (Schätzung der Kumulanten III. Ordnung [2]) und den im folgenden beschriebenen direkten Ansatz (Periodogramm III. Ordnung) [3]. Zunächst werden von jedem Trial i die Produkte der Fouriertransformierten gemäß (7) gebildet. Dabei kennzeichnet * die konjugiert komplexe Form.

$$
B_{i}\left(n_{1}, n_{2}\right)=X_{i}\left(n_{1}\right) \cdot X_{i}\left(n_{2}\right) \cdot\left(X_{i}\right)^{*}\left(n_{1}+n_{2}\right)
$$

Die Idee zum Übergang zu einer zeitvarianten Schätzung des Bispektrums besteht darin, die intervallbezogene klassische Fouriertransformation durch die zeitvariante, adaptiv rekursive Fouriertransformation zu ersetzen.

$$
\begin{aligned}
B_{i}^{c}\left(n_{1}, n_{2}, t\right)= & \operatorname{DFT}_{i}^{c}\left(n_{1}, t\right) \cdot \operatorname{DFT}_{i}^{c}\left(n_{2}, t\right) \\
& \cdot\left(D^{c} T_{i}^{c}\right)^{*}\left(n_{1}+n_{2}, t\right)
\end{aligned}
$$

Die Schätzung des Bispektrums ergibt sich schließlich aus der Mittelung über alle Trials.

$$
\hat{B}^{c}\left(n_{1}, n_{2}, t\right)=\frac{1}{K} \sum_{i=1}^{K} B_{i}^{c}\left(n_{1}, n_{2}, t\right)
$$

\section{Ergebnisse}

\section{Zeitvariante Spektralanalyse}

Die Funktionalität dieses Ansatzes wurde zunächst anhand der zeitvarianten Spektralanalyse II. Ordnung (Leistungsspektralanalyse) überprüft.

In der folgenden Abbildung ist das Ergebnis der Analyse eines Chirpsignals dargestellt. Bei einer Abtastfrequenz von $128 \mathrm{~Hz}$ steigt die Frequenz innerhalb von $5 \mathrm{~s}$ von $0 \mathrm{~Hz}$ auf $50 \mathrm{~Hz}$ an und nimmt in den darauffolgenden $5 \mathrm{~s}$ ebenso linear wieder ab. Die auf der Basis der ADITT gewonnene Zeit-Frequenz-Darstellung widerspiegelt diesen Frequenzverlauf.

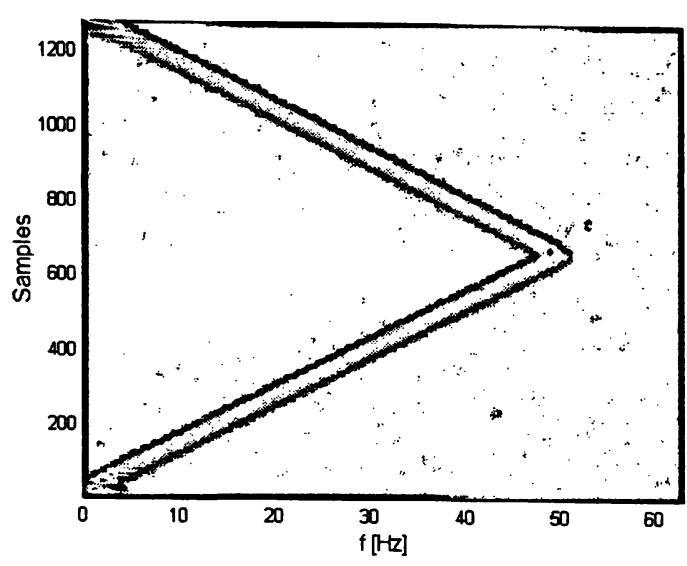

Abbildung 1: Zeit-Frequenz-Darstellung eines simulierten Chirp-Signals auf der Basis der ADFT $(c=0.03)$

In Abbildung 2 ist ein EEG-Signalabschnitt dargestellt, in dessen Verlauf der Proband die Augen schließt. Die zeitvariante Spektralanalyse mittels ADFT zeigt deutlich die in der zweiten Signalhälfte entstehende Leistung im AlphaFrequenzband $(8-13 \mathrm{~Hz})$.
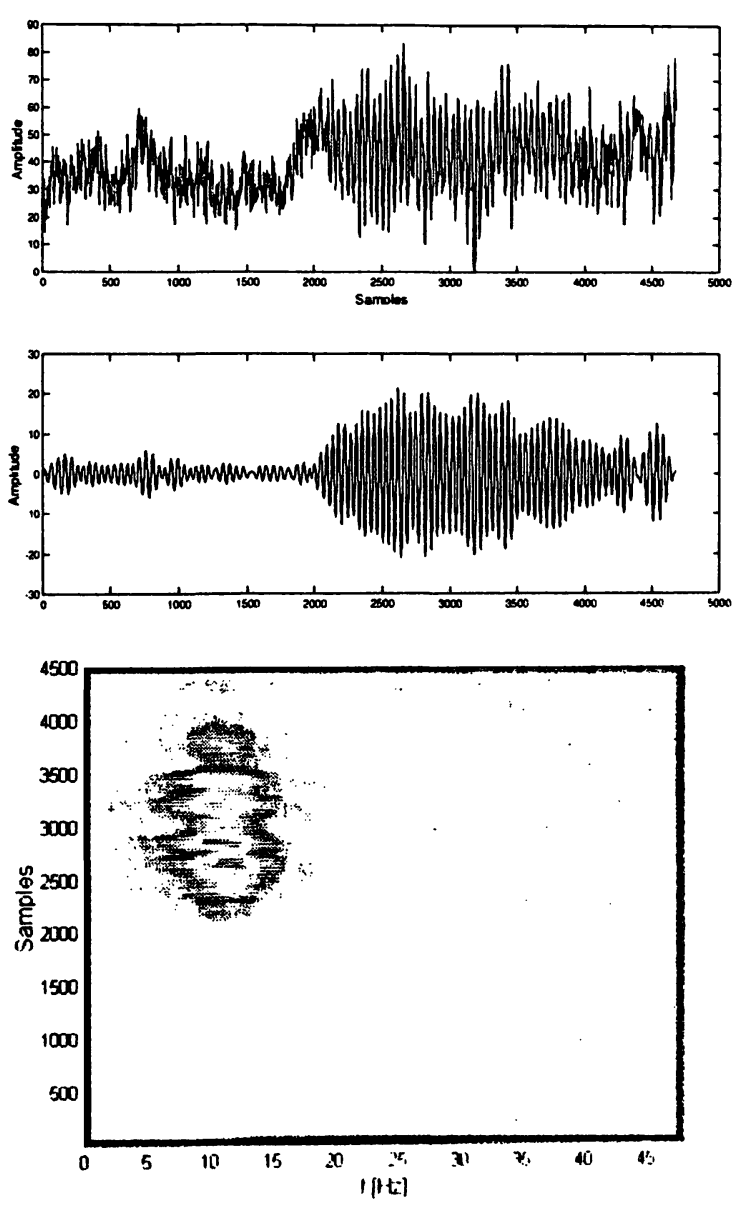

Abbildung 2: Zeitvariante ADIT-L.cistungmypehtral analyse eines EEG-Signalabschnittes coten: Rohlilici. Mittc: Alpha-Bandpassfiltening. unten: zellval, NIM:1. Spektrum) 
Analysce Iransienter quadratischer Phasenkopplungen Dic hauptsiichliche Motivation zur Entwicklung der ADFT ist dic Nutzung als Bassisalgorillumus für cine zcitvariante Bispektralanalyse. In den Abbildungen 3 und 4 sind erste Simulationsergebmisse dargestellt.

Am Bcispicl cines QI'C-Wechscls kann auch in Bispektrum die prinzipielle Funktionalitiit dieser Methode nachfewiesen werden. Jedech wirl ebenso deutlich, dass die AI)IPl-Schiilzung (Abb. 4 oben) gegenüber der Schäitzung auf der Basis der adaptiv rekursiven Kumulanten (Abb. 4 unten) cine wesentlich grölBere Varianz (schlechtere S('hiitzg(ite) bei gleicher Adaptionsvariable besitzt. Dieser Nachtcil macht sich im Bispektrum aut Grund der Produhthildung aus drei Fouriertransformierten gemäß (8) im Vergleich zuIn leistungsspektrum noch deutlicher benerhbir.
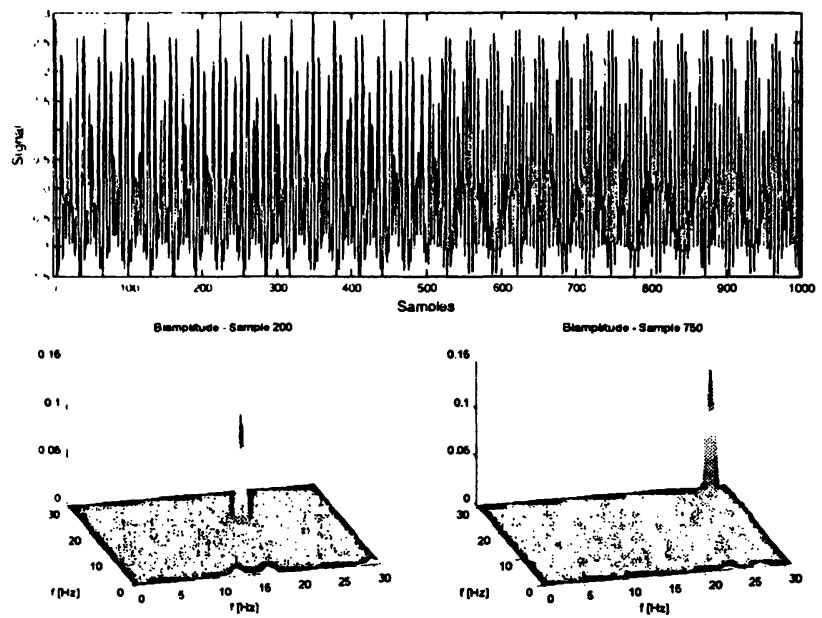

Abbildung 3: Signal mit QPC-Wechsel von 13/17/30 Hz $\rightarrow 23 / 27 / 50 \mathrm{~Hz}$ beim Sample $n=500$ (oben) und zeitvar. ADFT-Bispektrum ( $c=0.02$ ) für $n=200$ und $n=750$ (unten)
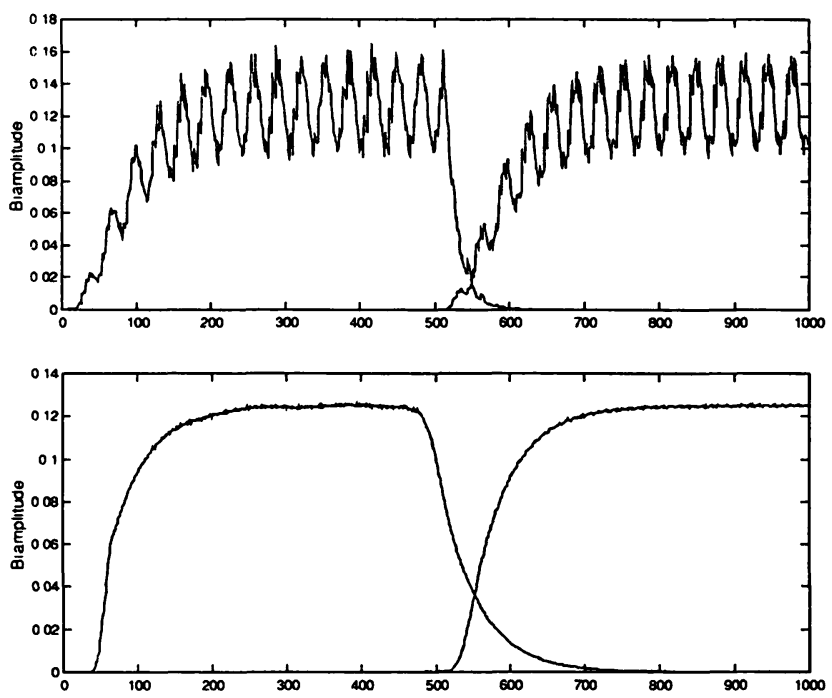

Abbildung 4: Zeitverläufe der Biamplituden der quadrat. phasengekoppelten Frequenzen $13 / 17 \mathrm{~Hz}$ (1. Hälfte) und $23 / 27 \mathrm{~Hz}$ (2. Hälfte); oben: ADFT-Schätzung, unten: mittels adaptiv rekursiver Schätzung der Kumulanten [2]

\section{Diskussion}

Iss wurdc eine neue Methode zur Schätzung des zeitvarianten Bispcktrums auf der Basis einer adaptiv rekursiven Fouricrtransformation vorgestellt und ihre Funktionsweise demonstricrt. Sic besitzt auf dem gegenwärtigen Entwicklungsstand gegenüber der bewährten zeitvarianten indirekten Schätzung [2] noch den Nachteil einer zu großen Varianz. In nachfolgenden Arbeiten muss untersucht werden, wie diese schlechtere Schätzgüte algorithmisch verbessert werden kann.

Andererseits bietet sie gegenüber der indirekten Schätzung wesentliche Vorteile. Zum einen ist man auf Grund des ADFT-Algorithmus nicht an die Berechnung der gesamten Bispektrummatrix gebunden. Es ist möglich, das Bispektrum für einzelne Frequenztripel (Bifrequenzpunkt) selektiv zu berechnen. Das ist ein entscheidender Vorteil für die Rechenperformance des Algorithmus. Zum anderen ist es möglich, den Algorithmus für jeden Bifrequenzpunkt separat mit einer eigenen Adaptionsvariablen zu steuern. Damit lässt sich z.B. die Varianz der Schätzung frequenzunabhängig gestalten. Dies ist beim indirekten Ansatz nicht möglich, da dort die gesamte Bispektrummatrix mit der gleichen Adaptionsvariablen berechnet wird. Außerdem erlaubt die ADFT, eine Normierung des komplexen Bispektrums gemäß den bekannten Verfahren nach Haubrich und $\mathrm{Kim} /$ Powers [3, S. 461-464] vorzunehmen (Bikohärenz), die im Falle indirekt geschätzter Bispektren zu numerischen Problemen führen [4].

Durch die zu Grunde liegenden einfachen Konstruktionsprinzipien ermöglicht auch die ADFT einen Einsatz im Echtzeitbetrieb. Im Gegensatz zu aus der Literatur bekannten Verfahren der Zeit-Frequenz-Darstellung existiert keine Bindung an ein Analyseintervall. Die Methode ist somit zur Untersuchung fortlaufender Signale geeignet.

\section{Danksagung}

Die Arbeit wurde gefördert durch die DFG-Projekte GR 1555/2-3 und WI 1166/2-3.

\section{Literaturverzeichnis}

[1] G. Grießbach, Computerorientierte Meßstochastik in der Technischen Diagnose und dem Signalmapping, Habilitationsschrift, FSU Jena, Math. Fakultät, 1990

[2] M. Helbig, G. Grießbach, B. Schack. H. Witte, „Application of time-variant bispectrum in biosignal analysis", Med. \& Biol. Eng. \& Comput., 37, Supplement 2, pp. 392-393, 1999

[3] C.L. Nikias, A.P. Petropulu, „Higher-order spectra analysis - a non-linear signal processing framework", New Jersey, PTR Prentice Hall, 1993

[4] M. Helbig, G. Grießbach, H. Witte, B. Schack, „Numerisch stabile Normierung des indirekt geschätzten Bispektrums“, Biomedizinische Technik, 46 (Ergänzungsband 1), pp. 318-319, 2001 